\title{
Energy Transportation by MeV Hot Electrons in Fast Ignition Plasma Driven with LFEX PW Laser ${ }^{*}$
}

\author{
Zhe ZHANG, Hiroaki NISHIMURA, Shinsuke FUJIOKA, Yasunobu ARIKAWA, Mitsuo NAKAI, \\ Hideo NAGATOMO, Hiroyuki SHIRAGA, Sadaoki KOJIMA, Alessio MORACE, \\ Noriaki MIYANAGA, Junji KAWANAKA, Yoshiki NAKATA, Takahisa JITSUNO, \\ Hiroshi AZECHI, Tetsuo OZAKI ${ }^{1)}$, Hui $\mathrm{CHEN}^{2)}$, Jaebum PARK ${ }^{2)}$, Gerald Jackson WILLIAMS ${ }^{2)}$, \\ Tomoyuki JOHZAKI ${ }^{3)}$ and Atsushi SUNAHARA ${ }^{4)}$ \\ Institute of Laser Engineering, Osaka University, 2-6 Yamada-oka, Suita, Osaka 565-0871, Japan \\ 1) National Institute for Fusion Science, LHD, High Temperature Plasma G. 322-6 Oroshi Toki, Gifu 509-5292, Japan \\ ${ }^{2)}$ Lawrence Livermore National Laboratory, Livermore, CA 94550, USA \\ ${ }^{3)}$ The Graduate School of Engineering, Hiroshima University, 1-4-1 Kagamiyama, Higashi Hiroshima 739-8527, Japan \\ 4) Institute for Laser Technology, 2-6 Yamada-oka, Suita, Osaka 565-0871, Japan
}

(Received 24 January 2014 / Accepted 13 May 2014)

\begin{abstract}
Implosion and heating experiments with the scheme of fast ignition (FI) have been performed on Gekko-XII and LFEX laser platform at the Institute of Laser Engineering in Osaka University. A cone-guided CD-shell has been used as a base-line target for the fast ignition realization. The information about energy transfer from LFEX petawatt (PW) laser is quantitatively studied with an absolute $K \alpha$ diagnostics and a fast electron trajectory simulation. The transfer efficiency $\left(\eta_{\mathrm{TE}}\right)$ is estimated for a planar interaction as a reference and for several types of guiding-cone. As a general trend, the guiding-cone enhanced the $\eta_{\mathrm{TE}}$ by a factor of 3 comparing with the planar case.
\end{abstract}

(C) 2014 The Japan Society of Plasma Science and Nuclear Fusion Research

Keywords: fast ignition, x-ray spectroscopy, laser transfer efficiency

DOI: $10.1585 /$ pfr.9.1404118

\section{Introduction}

Fast ignition is recognized as a promising pathway to efficient thermonuclear fusion in laser- driven inertial confinement fusion. A cone-guided CD-shell has been used as a base-line target for the fast ignition realization at Institute of Laser Engineering in Osaka University [1,2]. Experiments were taken out with Gekko-XII laser for implosions and LFEX laser for heating. It has long been expected to provide more quantitative information about the hot electron generation and transportation in the cone than those derived only with x-ray imaging and neutron detection. In this research, we propose an absolute $K \alpha$ line spectroscopy dedicated for quantitative measurement of hot electron generation and transportation in high- $Z$ targets. This diagnostic provides local information about the hot electrons propagating through specific materials composing the cone-guided target.

In this study, $\mathrm{Sn}$, Ta and $\mathrm{Au}$ were chosen as tracers since they are representative highest- $Z$ materials which are available for the guiding cone, thus better matching with $\mathrm{MeV}$-hot electrons than lower- $\mathrm{Z}$ tracers such as $\mathrm{Cu}$.

author'se-mail: zhang-z@ile.osaka-u.ac.jp

*) This article is based on the invited talk at the 30th JSPF Annual Meeting (2013, Tokyo).

\section{Absolutely $K \alpha$ Diagnostics}

A Laue spectrometer was developed to cover high energy $K \alpha$ lines from Mo ( $\left.K \alpha_{1}: 17.48 \mathrm{keV}, K \alpha_{2}: 17.37 \mathrm{keV}\right)$ to $\mathrm{Au}\left(K \alpha_{1}: 68.80 \mathrm{keV}, K \alpha_{2}: 66.99 \mathrm{keV}\right)$, as shown in Fig. 1 [3]. The spectrometer consists of a cylindrically curved quartz crystal $(10-11)$ plate and a detector. The quartz plate is bent such that the diffracted $\mathrm{X}$-rays are focused at an intermediate slit [4,5]. X-ray components propagating in a straightforward manner are shielded from the detector directly with a lead pinhole plate located in front of the crystal and a pair of lead shields located at the intermediate $\mathrm{x}$-ray focus. By varying the distances from

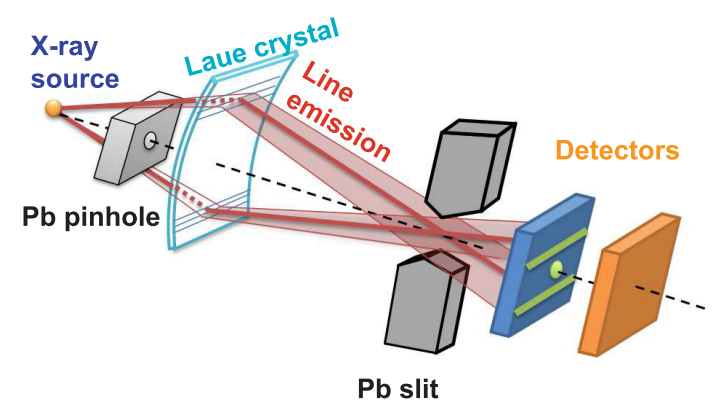

Fig. 1 The Laue spectrometer system. 


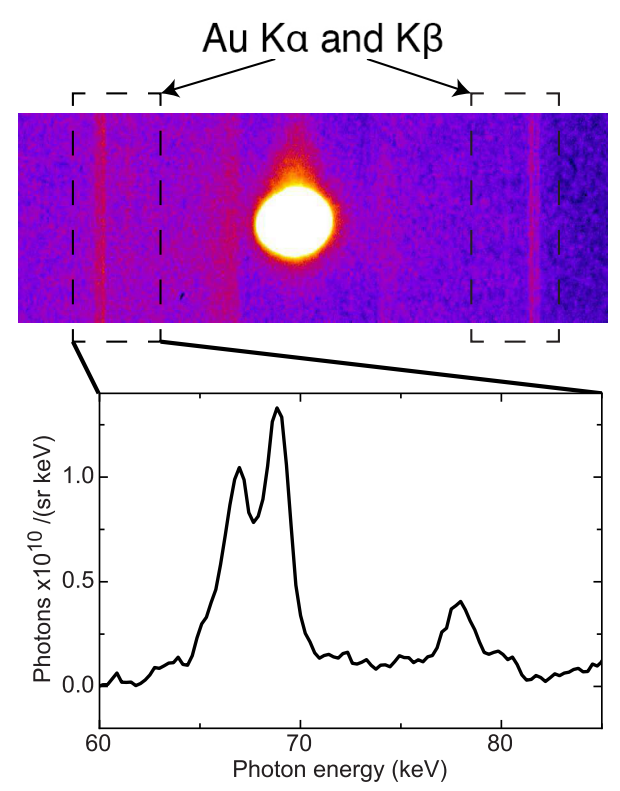

Fig. 2 The image from Au plasma on the Laue spectrometer.

the crystal to the source and detector, this spectrometer can cover the energy range of either $10-60 \mathrm{keV}$ or 22 $100 \mathrm{keV}$.

Figure 2 shows a typical image from the Au plasma. The bright spot in the center is the 0th order pinhole image and the line emission was imaged symmetrically on both sides. By plotting the line profile, the $K \alpha_{1}, K \alpha_{2}$ and $K \beta$ lines from Au were clearly seen.

\section{Hot Electron Trajectory}

The hot electron trajectory inside the solid target is tracked with a Monte-Carlo simulation. A 3-dimensional code PHITS was applied. The electrons propagation inside the target is treated with the continuous slowing down approximation (CSDA) model, which means that the electrons change their directions of motion due to elastic scattering, and lose their energy between two scattering points continuously. An example is shown in Fig. 3. The black square in the center indicates a $1 \mathrm{~mm}$ Ta cube, which is surrounded by low density air with a pressure of $10^{-5}$ Torr. The hot electron beam was irradiated on the Ta cube from the left side. An initial divergence of $45^{\circ}$ and a Gaussian spatial profile were assumed. The energy spectrum was set based on the electron spectrometer (ESM) measurement in experiments. From Fig. 3, it is clearly seen that most of the electrons with energy less than $0.1 \mathrm{MeV}$ were reflected at the front surface. The penetration depth increased along with electron energy; and only part of the electrons with energy higher than $1 \mathrm{MeV}$ can propagate through the $\mathrm{Ta}$ cube and escape from the back surface.

\section{Laser Transfer Efficiency}

The number of $K \alpha$ photons $N_{K \alpha}$ generated from hot electron with number $N_{\mathrm{h}}$ and temperature $T_{\mathrm{h}}$ can be esti-

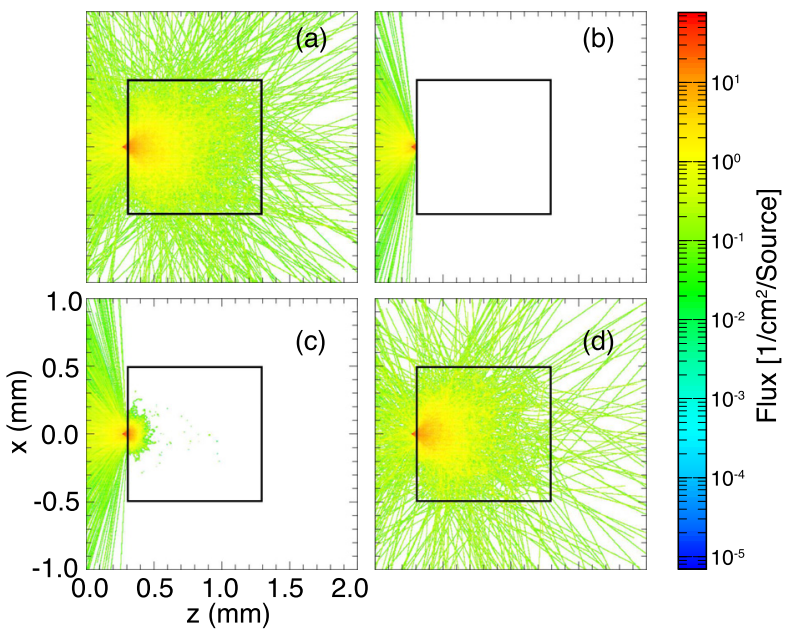

Fig. 3 Electron trajectory in the Ta cube: (a) all electrons, (b) electron with energy less than $0.1 \mathrm{MeV}$, (c) electron with energy from 0.1 to $1 \mathrm{MeV}$, (d) electron with energy more than $1 \mathrm{MeV}$.

mated by the following model [6]:

$$
\begin{aligned}
N_{K \alpha}= & N_{\mathrm{h}} \frac{n_{\mathrm{A}} \omega_{K \alpha}}{4 \pi} \int_{0}^{\infty} d E \sigma_{K \alpha}(E) \times \\
& \int_{0}^{d} d x f_{\mathrm{h}}\left(E_{0}, x\right) \exp \left(-\frac{x}{\lambda_{\mathrm{mfp}} \cos (\theta)}\right),
\end{aligned}
$$

where $\sigma_{K \alpha}, \omega_{K \alpha}$, and $n_{\mathrm{A}}$ are, respectively, the cross section for K-shell ionization, the $K \alpha$ fluorescence yield, and the atomic number density. The term $\exp \left(-\frac{x}{\lambda_{\operatorname{mfp}} \cos (\theta)}\right)$ describes the reabsorption of $K \alpha$ photons during the propagation through the target material, where $\theta$ is the angle between the spectrometer and the target normal. $f_{\mathrm{h}}\left(E_{0}, x\right)$ describes the energy spectrum for the hot electron propagating inside the Ta cube with a depth $x$, where the information was achieved with the Monte-Carlo simulations. At $x=0$, the hot electron energy spectrum has an one or two temperature exponential distribution as:

$$
f_{\mathrm{h}}\left(E_{0}\right)=N_{\mathrm{h} 1} \times \exp \left(-\frac{E}{T_{\mathrm{h} 1}}\right)+N_{\mathrm{h} 2} \times \exp \left(-\frac{E}{T_{\mathrm{h} 2}}\right),
$$

where $N_{\mathrm{h} 1}$ and $N_{\mathrm{h} 2}$ represent the number of hot electron with temperature $T_{\mathrm{h} 1}$ and $T_{\mathrm{h} 2}$. Considering the absolute $K \alpha$ photon number $N_{K \alpha}(\exp )$ measured by the Laue spectrometer, the transfer efficiency $\eta_{\mathrm{TE}}$ can be estimated by comparing the experimental measurement with simulation results as:

$$
\eta_{K \alpha}=\frac{\frac{N_{K \alpha}(\exp )}{N_{K \alpha}(\operatorname{sim})} \times \int_{0}^{\infty} f_{\mathrm{h}}\left(E_{0}\right)(\operatorname{sim}) d E}{E_{\mathrm{laser}}} .
$$

The transfer efficiency from LFEX laser to target has been estimated in the cases of planar and cone-guided geometries. In the case of planar target, an Au plate was placed at the target chamber center and LFEX laser with 


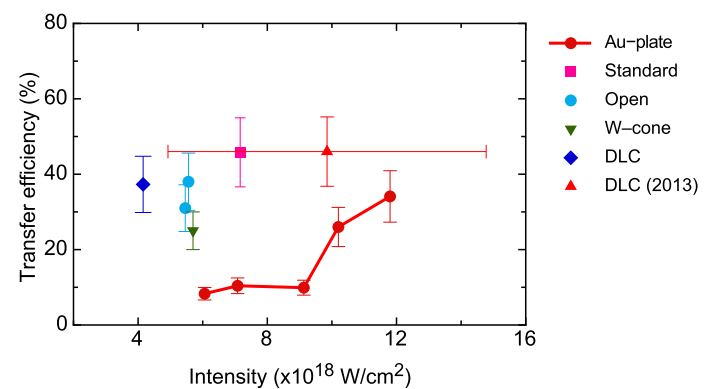

Fig. 4 The $\eta_{\text {TE }}$ as a function of laser intensity for the planar and cone-guided targets. DLC(2013) is the DLC cone attached with a double-layer target.

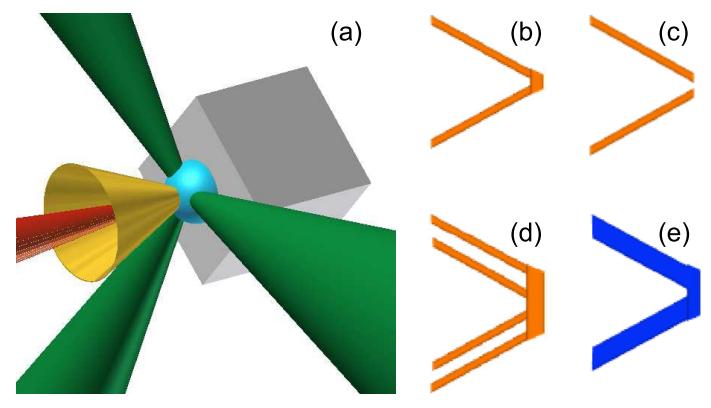

Fig. 5 (a) The configuration of a cone $+\mathrm{CH}$ hemi shell+Ta cube target; (b) Standard Au cone; (c) Open Au cone; (d) Wcone; (e) DLC cone.

maximum energy of $1.8 \mathrm{~kJ}$ was focused on the front surface with an incident angle of $10^{\circ}$. The $K \alpha$ from Au was recorded by the Laue spectrometer and an ESM was located from the back surface for the hot electron temperature. The $\eta_{\mathrm{TE}}$ as a function of laser intensity is shown in Fig. 4. As a general trend, the $\eta_{\mathrm{TE}}$ is increased by increasing the laser intensity.

The cone-guided configuration is shown in Fig. 5. A cone is attached with a $\mathrm{CH}$ hemi-shell, which was irradiated by three beams of Gekko-XII laser [2]. A dense plasma surrounding the tip of the cone was produced to mimic the condition of the fast ignition Au cone+CD shell target. The Ta cube was attached as the $K \alpha$ tracer after the $\mathrm{CH}$ hemi-shell. Four types of cone were used, as shown in Figs. 5(b)-(e): (b) is the standard Au cone with $7 \mu \mathrm{m}$ thickness; (c) is the Au open-cone without the tip; (d) is the W-cone with double Au layers; and (e) is the diamond like carbon (DLC) cone. The estimated $\eta_{\mathrm{TE}}$ was shown in Fig. 4.

\section{Double-Layer Target}

In order to achieve more information about the low energy hot electrons rather than that derived only from ESM, a double-layer target has been designed and tested experimentally. The basic idea is to add a second $K \alpha$ tracer layer in front of the Ta. Tentatively, a $200 \mu \mathrm{m}$ Sn was chosen:

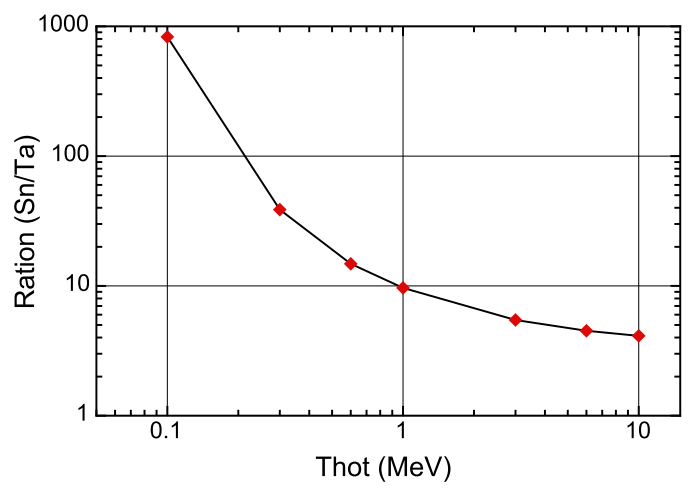

Fig. 6 The ratio of $\mathrm{Sn}$ to Ta $K \alpha$ photon numbers as a function of effective hot electron temperature.

firstly, the $K \alpha$ lines from $\mathrm{Sn}$ can be clearly separated from Ta in the Laue spectrometer; secondly, the electrical resistivity of $\mathrm{Sn}$ is very similar to Ta, which can suppress the hot electron current change between the intermediate surface. The ratio of Sn to Ta $K \alpha$ photon numbers was simulated as a function of the effective hot electron temperature as shown in Fig. 6. This ratio is very sensitive for low temperature hot electrons. One of this double-layer target was experimentally tested with DLC cone. The ratio of Sn to Ta $K \alpha$ photon number was measured with Laue spectrometer as 4.6. From Fig. 6, the effective hot electron temperature is $5.3 \mathrm{MeV}$. Considering the quantitative yield of $\mathrm{Sn}$ and Ta $K \alpha$ photons, the $\eta_{\mathrm{TE}}$ was estimated to be about $46 \%$, as plotted in Fig. 4.

\section{Conclusion}

$K \alpha$ line spectroscopy, particularly for hard x-ray region, has been proposed for quantitative measurement of cone-guided fast ignition experiment. The Sn, Ta and Au $K \alpha$ lines were observed and the energy transfer efficiency was provided. Compared with the planar geometry, the LFEX laser transfer efficiency is significantly enhanced with a guiding cone. Besides ESM, a double-layer target was designed to achieve more information about the low energy hot electrons. The $K \alpha$ photon numbers from the two tracer materials were used to estimate the effective hot electron temperature. In the near future, the thickness and materials of the double-layer target will be further optimized.

\section{Acknowledgments}

The authors would like to thank the Gekko-XII and LFEX laser operation crew, the target fabrication group, the plasma diagnostics group, and the computer operation staffs for their great contribution to this work. The work by LLNL staffs were performed under the auspices of the U.S. DOE by LLNL under Contract DE-AC52-07NA27344. 
[1] R. Kodama et al., Nature 412, 6849 (2001).

[2] H. Shiraga, S. Fujioka, M. Nakai et al., Plasma Phys. Control. Fusion 53, 124029 (2011).

[3] Z. Zhang, H. Nishimura, T. Namimoto et al., Rev. Sci. Instrum. 83, 053502 (2012).
[4] Y. Cauchois, J. de Phys. et le Radium 3, 320 (1932).

[5] J.F. Seely, L.T. Hudson, G.E. Holland and A. Henins, Appl. Opt. 47, 2767 (2008).

[6] D. Salzmann, C. Reich, I. Uschmann, E. Förster and P. Gibbon, Phys. Rev. E 65, 036402 (2002). 